\title{
X-RAY EMISSION FROM THE GALACTIC DISK
}

\author{
R. D. BLEACH \\ E. A. BOLDT \\ S. S. HOLT \\ D. A. SCHWARTZ \\ P. J. SERLEMITSOS
}

MARCH 1972

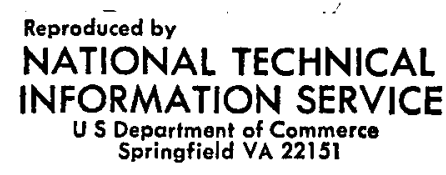

GSFC

GODDARD SPACE FLIGHT CENTERT

GREENBELT, MARYLAND

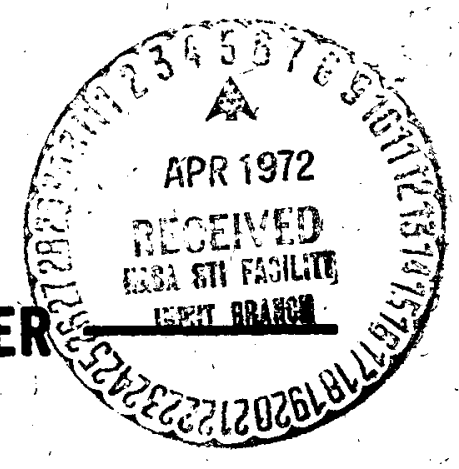

(NASA-TH-X-65857) X-RAY EMISSION FROM THE

GALACTIC DISK R.D. Bleach, et aI (NASA)

[lar. $197215 \mathrm{p}$

CSCL O3B

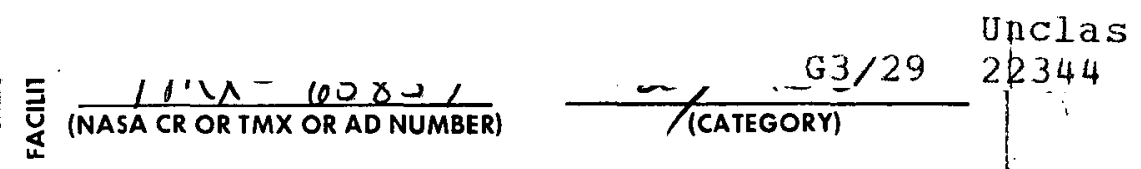




\section{X-RAY EMISSION FROM THE GALACTIC DISK}

R. D. Bleach*, E. A. Boldt, S. S. Holt, D. A. Schwartz**, and P. J. Serlemitsos

NASA/Goddard Space Flight Center

Greenbe1t, Maryland

\section{ABSTRACT}

A search was made for a diffuse component of $\mathrm{X}$-rays $>1.5 \mathrm{keV}$ associated with an interarm region of the galaxy at galactic longitudes in the vicinity of 60 degrees. A statistically significant excess associated with a narrow disk component was detected. The angular extent of this component has a most probable value of 2 degrees and may be as large as 7 degrees at $90 \%$ confidence. The best $\mathrm{fit}$ spectrum yields an intensity of 2.9 photons $\left(\mathrm{cm}^{2}-\mathrm{sec}-\mathrm{ster}\right)^{-1}$ over the 2 - $10 \mathrm{keV}$ range. The 30 upper limit to any emission (e.g. iron line) in a $1.5 \mathrm{keV}$ band centered at $7 \mathrm{keV}$ from galactic latitudes $|\mathrm{b}| \leq 3.5$ degrees is .3 photons $\left(\mathrm{cm}^{2}-\mathrm{sec-ster}\right)^{-1}$. Severa 1 possible emission models are discussed, with the most likely candidate being a population of unresolvable low luminosity discrete sources.

Permanent Address: Dept. of Physics, University of Maryland, College Park, Md.

NAS-NRC Resident Research Associate

Present Address: American Science and Engineering Cambridge, Mass. 


\section{The Experiment}

Two multi-anode, multi-layer proportional counters were flown on August 9, 1971 aboard an Aerobee 170 rocket launched from White Sands, New Mexico. Each detector had a $1.8^{\circ} \times 7.1^{\circ}$ (FWHM) mechanical rectangular collimator with $650 \mathrm{~cm}^{2}$ net area. The detector filled with P10 ( $90 \%$ argon, $10 \%$ methane) was sensitive to $1.5-20 \mathrm{keV}$ photons and the detector with a xenon-methane mixture in similar proportions was sensitive to $2-40 \mathrm{keV}$ photons. Each detector had an entrance window of 1 mil aluminized Kapton. The aspect solution, obtained from two cameras, provided positional accuracy estimated to be about .2 degrees.

The detectors were oriented with their $7.1^{\circ}$ collimation parallel to the galactic plane as they scanned galactic longitudes centered from 52 through 61.8 degrees ( $a t b=-.5$ degrees) with a yaw rate of approximately .5 degrees per second. A roll program was initiated at galactic longitude 61.8 degrees during which the detectors made 4 scans back and forth across the plane in the 1.8 degree collimation direction with a half-amplitude of about 7.5 degrees, centered at $b=-.5^{\circ}$.

The data discussed in this paper were obtained during 126 seconds of the yaw and roll portion of the flight with an additional 16 seconds of diffuse $x$-ray data collected at galactic latitudes $|\mathrm{b}| \geq 8^{\circ}$. Spatial Profile

Data from four successive scans across the galactic plane along the 1.8 degree collimation direction at longitude 61.8 degrees, folded into 1 degree bins in galactic latitude, are shown in Figure 1 . These data were obtained from a portion of the galactic plane scanned in longitude during a previous flight of the same payload on September 21,1970 
(Schwartz et al., 1971). No discrete source was observed above $2 \mathrm{keV}$ with a $99 \%$ confidence for a flux of .03 photons $\left(\mathrm{cm}^{2}-\mathrm{sec}\right)^{-1}$ anywhere in the region $45^{\circ} \leq \ell \leqslant 70^{\circ}$, or .02 photons $\left(\mathrm{cm}^{2}-\mathrm{sec}\right)^{-1}$ as a $3 \sigma$ upper limit to a source at any given position in the same region. In addition, there are two measurements of the diffuse intensity at $\left|b^{\prime}\right| \geq 8^{\circ}$ which are included in the profile: The average counting rate during a 20 second interval when the detectors were pointed at the earth was $8.6 \mathrm{sec}^{-1}$. The data of Figure 1 were found to be inconsistent with a constant counting rate to a $x^{2}$ confidence level exceeding $99 \%$ percent. We have also attempted a least squares fit to the profile shown in Figure 1 using a two parameter point source model of the form

$$
\mathbf{N}_{\mathbf{i}}=\mathbf{A}+\mathrm{Bf}_{\mathbf{i}}
$$

where:

$$
\begin{aligned}
N_{i}= & \text { total counts in the } i \text { th } b i n \\
A= & \text { diffuse non-disk background counts in the } i_{\text {th }} \text { bin } \\
B= & \text { total observed source counts } \\
f_{i}= & \text { fraction of total counts from a point source } \\
& \text { expected in the } i \text { th bin }
\end{aligned}
$$

For point sources of constant strength located at $b=0$ degrees, this model can be excluded to a $x^{2}$ confidence level of $92 \%$, while point sources at any other single latitude give a poorer fit.

Recently, however, the UHURU satellite has detected a discrete variable $X$-ray source at $\ell=68.45$ degrees and $b=1.68$ degrees with a maximum strength of about 12 percent that of Cygnus X-2 (c.f. Giacconi et al., 1971). During our previous rocket flight mentioned above, 
this source should have been observable at a strength of approximately 20 percent of the maximum reported from UHURU, but was not seen. We have attempted a least squares fit of the form given by Equation 1 to a source at that location and find that it can be rejected at a $x^{2}$ confidence exceeding 99 percent. A discrete source at the position and maximum strength observed by UHURU wonld contribute about 4 counts/sec。 to the $b=1-2$ degree bin of Figure 1 .

With regard to possible $\mathrm{X}$-ray emission from an extended object such as that seen by Katgert (1969) at $21 \mathrm{~cm}$. having its center at galactic coordinates $\mathrm{b}=-.3$ degrees, $\ell=61.5$ degrees, we can rule out any major contribution to our effect from this object on the basis of our September 1970 flight (c.f. Schwartz et al., 1972). We would have observed this object while scanning it in galactic longitude at almost the same sensitivity as in the current flight in which it was scanned in galactic latitude. Since the object is believed to have arisen from multiple supernova explosions and has an estimated age of about $10^{6}$ years, it seems likely that any hard X-ray emission would have long since disappeared.

A uniformly emitting disk mode1 was least squares fitted to the data giving acceptable results for disks with angular extent up to 7 degrees based on $90 \%$ confidence limits of the $x^{2}$ distribution. The best fitting model of angular extent' 2 degrees gives the intensity from the disk as 2.9 photons $\left(\mathrm{cm}^{2}-\mathrm{sec}-\mathrm{ster}\right)^{-1}$ over a $2-10 \mathrm{keV}$ energy range for the galactic interarm region $\left(7.1^{\circ} \mathrm{FWHM}\right)$ centered at $l=61.8^{\circ}$. We note, however, that the average gross counting rate during the initial yaw in galactic longitude (at $\mathrm{b}=-.5^{\circ}$ ) over $l=52^{\circ}-61.8^{\circ}$ is about $20 \%$ 
higher than that inferred for $b=0^{\circ}$ from the 4 roll scans (at $\ell=61.8^{\circ}$ ) previously mentioned, an effect which could be related to galactic structure.

Spectrum

The net disk spectrum was derived by subtracting the data in Figure 1 which were obtained with the collimator centered at $|\mathrm{b}|>3.5$ degrees from data where $|\mathrm{b}| \leq 3.5$ degrees. Figure 2 shows the spectrum of the net incident intensity renormalized to $b=0$ degrees for the best fit disk. The best fit spectrum was obtained by comparison of the data with power law and exponential shapes for the source function (q), assuming a uniformly emitting disk of angular extent $\theta$ as follows:

$$
I_{b}=\frac{1}{4 \pi} \int_{0}^{N} \frac{q}{n} e^{-\mu r} d r+I_{B} e^{-\mu N}
$$

\section{$\frac{\text { photons }}{\mathrm{cm}^{2}-\text { sec-ster-keV }}$}

where $I_{b}=$ disk spectrum at galactic latitude $b$

$$
\begin{aligned}
I_{B}= & \text { isotropic extragalactic background spectrum } \\
q= & \text { photon source function }\left(\mathrm{cm}^{3}-\mathrm{sec}-\mathrm{keV}\right)-1 \\
\mathrm{n}= & \text { interstellar hydrogen density (H atoms } \left./ \mathrm{cm}^{3}\right) \\
\mu= & \text { absorption cross-section due to interstellar gas } \\
\mathrm{N}= & \text { columnar density (H atoms } \left./ \mathrm{cm}^{2}\right) \text { of the emitting region } \\
& \text { along the Iine of sight given by } \mathrm{N}=\text { constant for } \\
& |\mathrm{b}| \leq \frac{\theta}{2} \text { and } \mathrm{N} \propto \mathrm{csc}|\mathrm{b}| \text { for }|\mathrm{b}| \geq \frac{\theta}{2}
\end{aligned}
$$

Using the Brown and Gould (1970) absorption cross-section, with the addition of an iron abundance $3.1 \times 10^{-5}$ relative to hydrogen (Nussbaumer and Swings, 1970), we find that our photon source function can be 
expressed as

$$
\mathrm{q}=\left(8.5^{+3.8}-1.9\right) \times 10^{-21} \mathrm{E}^{-(2.8 \pm .5)} \frac{\text { photons }}{\mathrm{cm}^{3}-\mathrm{sec}-\mathrm{keV}}
$$

for a power law fit and

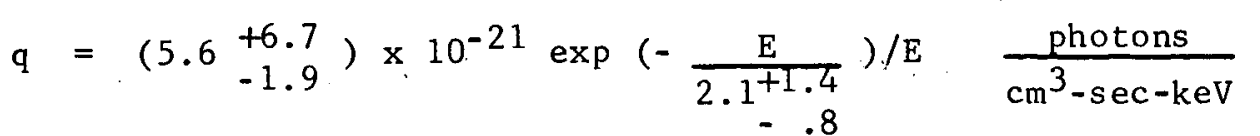

for an exponential fit. These values are based on an average interstellar hydrogen density of $.7 \mathrm{H}$ atoms $/ \mathrm{cm}^{3}$, and 1 imits are determined by using, $\mathrm{N}=(2 \pm 1) \times 10^{22} \mathrm{H}$ atoms $/ \mathrm{cm}^{2}$ from $21 \mathrm{~cm}$ data (Daltabuit, 1970; Burton, 1970). Both exponential and power law models gave about the same goodness of fit. We can also set a $3 \sigma$ upper limit of .3 photons $\left(\mathrm{cm}^{2} \text {-sec-ster }\right)^{-1}$ to any emission within a $1.5 \mathrm{keV}$ bin centered at $7 \mathrm{keV}$ (e.g. iron line) from the galactic disk at latitudes $|\mathrm{b}| \leq 3.5$ degrees. Such narrow band emission could be produced by charge exchange between stopping energetic iron nuclei and neutral $\mathrm{H}$ atoms, resulting in the ultimate capture of electrons to the $K$ shell (Silk and Steigman, 1969). If we assume that every iron atom now in the interstellar gas emitted two $\mathrm{K}$ x-rays (c.f. Ramaty et a1., 1971) within the galactic lifetime ( 10 10 years), then the time averaged line intensity would be comparable to this upper 1 imit.

\section{Discussion}

Several mechanisms have been formulated to explain the galactic excess emission examined by other observers (c.f. Cooke et a1., 1969; Schwartz 1969; Bunner et a1., 1971; Hayakawa et a1., 1970; Bleeker and Deerenberg, 1970). Inverse compton radiation from far infra-red photons 
has been discussed by Cowsik and Pal (1969), Shen (1969), O'Connell and Verma (1969), and Ipavich and Lenchek (1970). Such a process would produce a photon power law spectrum with an index of about 1.8 if the directly observed electron spectrum in the $5-200 \mathrm{GeV}$ range given by Anand et al. (1968) is used and 1.4 if low frequency $\left(<10^{2} \mathrm{MHz}\right.$ ) radio synchrotron measurements of galactic electrons in the $200 \mathrm{MeV}$ to a few GeV range (Alexander et al., 1969; Goldstein et al., 1970) are used, as contrasted with our observed spectral index of $2.8 \pm .5$. Using either electron spectrum (with the normalization of Goldstein et al. (1970) for the 1.4 index spectrum) the inverse Compton flux predicted from $2.70 \mathrm{~K}$ blackbody radiation is less than 5 percent of our measured flux. We cannot, however, exclude additional contamination arising from submillimeter photons of energy density equal to the upper limit of $4.3 \mathrm{eV} / \mathrm{cm}^{3}$ set by Hudson et al. (1971).

Synchrotron radiation losses from galactic electrons in the interstellar magnetic field (Freitas Pachaeco, 1970) could explain the observed flux if the cosmic ray electron spectrum observed at several hundred $\mathrm{GeV}$ is extrapolated to about $10^{6} \mathrm{GeV}$. The photon number spectral index of about 1.8 produced by such electrons, however, is incons istent with our limits on spectral shape. If we allow for a break of unity in the electron spectral index at $10^{3} \mathrm{GeV}$ corresponding to a galactic confinement 1 ifetime of about $10^{6}$ years for uniformly produced electrons, we find consistency with our observed spectral shape but with a photon intensity amounting to only a few percent of that observed. Upper limits given by Kamata et al. (1968) on $10^{4}-10^{5} \mathrm{GeV}$ electrons from a ir shower measurements were used to calculate a synchrotron intensity which is an 
order of magnitude below our observations. A spectral break of unity in the interstellar electron spectrum at about $6 \times 10^{4} \mathrm{GeV}$ could account for our results via the synchrotron mechanism, but we have no reason for expecting such a break. The radiative lifetime at this energy is about $1.5 \times 10^{4}$ years, during which time we would expect $<10^{-1}$ galactic supernovae in our field of view.

The collision of suprathermal charged particles with ambient free and atomic electrons has been suggested by Boldt and Serlemitsos (1969) as a means of producing $\mathrm{X}$-ray photons associated with the galactic disk. This interpretation of our data, however, requires an ionization rate $28 \times 10^{-14}$ ionizations ( $\mathrm{sec}-\mathrm{H}$ atom) ${ }^{-1}$ which is more than an order of magnitude greater than rates calculated by Hjellming et al. (1969) using pulsar dispersion measures and about an order of magnitude greater than the results of $\mathrm{H} \beta$ optical recombination measurements made by Reynolds et a1. (197l).

We have investigated transition radiation as a means of explaining our effect, in the light of a recent article by Johanssen (1971) suggesting that relativistic charged particles passing through interstellar grains could produce hard X-rays. Ramaty and Bleach (1972) have found, however, that because of the small size of the grains (about $10^{-5} \mathrm{~cm}$ ) transition radiation could not be produced in any significant quantity above photon energies on the order of $100-200 \mathrm{eV}$. A galactic excess above $2 \mathrm{keV}$ is therefore incompatible with such a model. We note that an extrapolation of our steepest fit to a power law source function down to $.26 \mathrm{keV}$ would yield an apparent intensity which is only $6 \%$ of the soft X-ray galactic component observed by Bunner et al. (1971). 
The result of many unresolved low luminosity discrete sources is capable of producing the effect we observe. Assuming that this sample is composed of sources of a single luminosity, then there must be a sufficient number in order to justify the use of our source function (q) throughout the disk. For objects with intrinsic luminosities greater than about $10^{35} \mathrm{ergs} / \mathrm{sec}$, our integral source function of about $7 \times 10^{-30}$ ergs $/ \mathrm{cm}^{3}-\mathrm{sec}(2-10 \mathrm{keV})$ implies that less than $10^{3}$ such sources could comprise a disk population. Our detectors have sufficient sensitivity, however, to measure all of these sources as discrete.

A galactic model has been suggested by Ryter (1970) in which the disk is composed of resolved discrete $X$-ray sources out to a distance (d) in the plane of the disk and unresolved sources of the same luminosity class at distances greater than $d$. When applied to our results, this model yields a distance (d) equal to 80 percent of the disk's extent in the plane. Since the angular extent of the disk using the above distance is approximately the same for both resolved and unresolved sources we have attempted to compare the angular size of our disk with a disk composed of already resolved sources from the UHURU catalog (c.f. Giacconi et al, 1971). The resolved galactic sources found in the region $30^{\circ} \leq \ell \leq 330^{\circ}$, excluding sources of strength $>10^{-1}$ Tau $X-1$, would produce an effective disk brightness with angular extent $\gtrsim 10$ degrees based on absolute values of galactic latitude for source positions. Since this result exceeds our $90 \%$ confidence limit to the angular extent of the disk, we conclude that unresolved $X$-ray sources with intrinsic luminosities similar to previously resolved low latitude sources probably cannot by themselves adequate ly describe our effect. 
Our upper limit of 7 degrees for the angular extent of the diffuse emission corresponds to a galactic disk thickness of $1.2 \mathrm{kpc}$ for a distance to the edge of the plane equal to $10 \mathrm{kpc}$. We note that the UHURU catalog (Giacconi et al., 1971) includes isotropically distributed objects of relatively weak flux, some of which might be low luminosity sources within the galaxy. The limiting sensitivity of UHURU to sources as distant as $0.6 \mathrm{kpc}$ corresponds to an intrinsic luminosity of $\sim 10^{33}$ ergs/sec. In this case, when UHURU completes a full sky survey with its limiting sensitivity, it should detect an isotropic distribution of $\leq 60$ sources of intrinsic luminosity $\leq 10^{33} \mathrm{ergs} / \mathrm{sec}$, while the unresolved sources of the same population in the disk at distances greater than $0.6 \mathrm{kpc}$ could produce our observed excess. Acknowledgments

It is a pleasure to acknowledge F. Birsa and C. Glasser for their technical support in carrying out this experiment and R. Ramaty for discussions. We also thank the sounding rocket group at Goddard Space Flight Center and the launch crew at White Sands Missile Range for their cooperation. 


\section{References}

Alexander, J. K., Brown, L. W., Clark, T. A., Stone, R. G., 1970, Astron. and Astrophysics, $6,476$.

Anand, K. C., Danie 1, R. R., and Stephens, S. A. 1968, Phys. Rev. Letters, 20, 764 .

Bleeker, J. A. M., and Deerenberg, A. J. M. 1970, Nature, 227, 470 .

Boldt, E. A., and Serlemitsos, P. J. 1969, Ap. J., 157, 557.

Brown, R., and Gould, R. 1970, Phys. Rev., D1, 2252.

Bunner, A. N., Coleman, P. L., Kraushaar, W. L., and McCammon, D. 1971, Ap. J. (Letters), 167, L3.

Burton, W. B. 1970, Astron. and Astrophysics, Suppl. 2, 261.

Cooke, B. A., Griffiths, R. E., and Pounds, K. A. 1969, Nature, 224, 134.

Cowsik, R., and Pal, Y. 1969, Phys. Rev. Letters, 23, 1467.

Daltabuit, E. 1970, preprint.

Freitas Pacheco, J. A. de 1970, Astron. and Astrophysics, $\underline{8}, 420$.

Giacconi, R., Gursky, H., Kellogg,. E., Murray, S., Schreier, E., and Tanabaum, H. 1971, (A.S.E. preprint 非855), submitted to Ap. J..

Goldstein, M. L., Ramaty, R., and Fisk, L. A. 1970, Phys. Rev. Letters, 24, 1193 .

Hayakawa, S., Kato, T., Makino, F., Ogawa, H., Tanaka, Y., and Yamashita, K. Non Solar X and Gamma Ray Astronomy, L. Gratton, editor (Dordrecht: D. Reidel Publishing Co., 1970).

Hjellming, R. M., Gordon, C. P., and Gordon, K. J. 1969, Astron. and Astrophysics, 2, 202.

Hudson, H. S., Peterson, L. E., and Schwartz, D. A. 1971, Nature, 230,177 .

Ipavich, F. and Lencheck, A. 1970, Phys. Rev., D2, 266.

Johanssen, S. A. E. 1971, Astrophysical Letters, 9, 143. 
Kamata, K., Shibata, S., Saavedra, O., Domingo, V., Suga, K., Murakami, K., Toyoda, Y., LaPointe, M., Gaebler, J., and Escobar, I. 1968, Can. Journ. Phys., 46, S72.

Katgert, P. 1969, Astron. and Astrophysics, 1, 54.

Nussbaumer, H. and Swings, J.P. 1970, Astron. and Astrophys., I, 455.

O'Conne11, R. F., and Verma, D. S. 1969, Phys.'Rev. Letters, 22, 1443.

Ramaty, R., Boldt, E. A., Colgate, S. A., and Silk, J. 1971, Ap. J., $169,87$.

Ramaty, R., and Bleach, R. D. 1972, (submitted to Astrophysical Letters).

Reynolds, R., Roesler, F., Scherb, F., and Boldt, E. 1971, The Gum. Nebula and Related Problems (ed. S. P. Maran, J. C. Brandt, T. P. Stecher), GSFC X-683-71-375.

Ryter, C. 1970, Astron. and Astrophysics, 9, 288.

Schwartz, D. A. 1969, Ph.D. Thesis, Univ. of California, San Diego.

Schwartz, D. A., Boldt, E. A., Holt, S. S., Serlemitsos, P. J., and Bleach, R. D. 1971, Nature, 233, 110.

Schwartz, D. A., Bleach, R. D., Boldt, E. A., Holt, S. S., and Serlemitsos, P. J. 1972 (to be published in April 15 Ap. J. Letters).

Shen, C. S., 1969, Phys. Rev. Letters, 22, 568.

Silk, J., and Steigman, G. 1969, Phys. Rev. Letters, 23, 597. 


\section{Figure Captions}

Figure 1. Top: Spatial profile $(2-10 \mathrm{keV})$ of galactic disk (4 scans summed) at $\ell=61.8^{\circ}$ plus two data bins from $b \leq-8^{\circ}$. Dotted line represents best fit to point source plus background. Bottom: Same data as above. Dotted line represents best fit to disk model plus background.

Figure 2. Incident galactic disk spectrum at $\mathrm{b}=0^{\circ}$. 
FIgURE 1 -

POINT SOURCE FIT TO GALACTIC PLANE PROFILE $\left(\ell=61.8^{\circ}\right)$

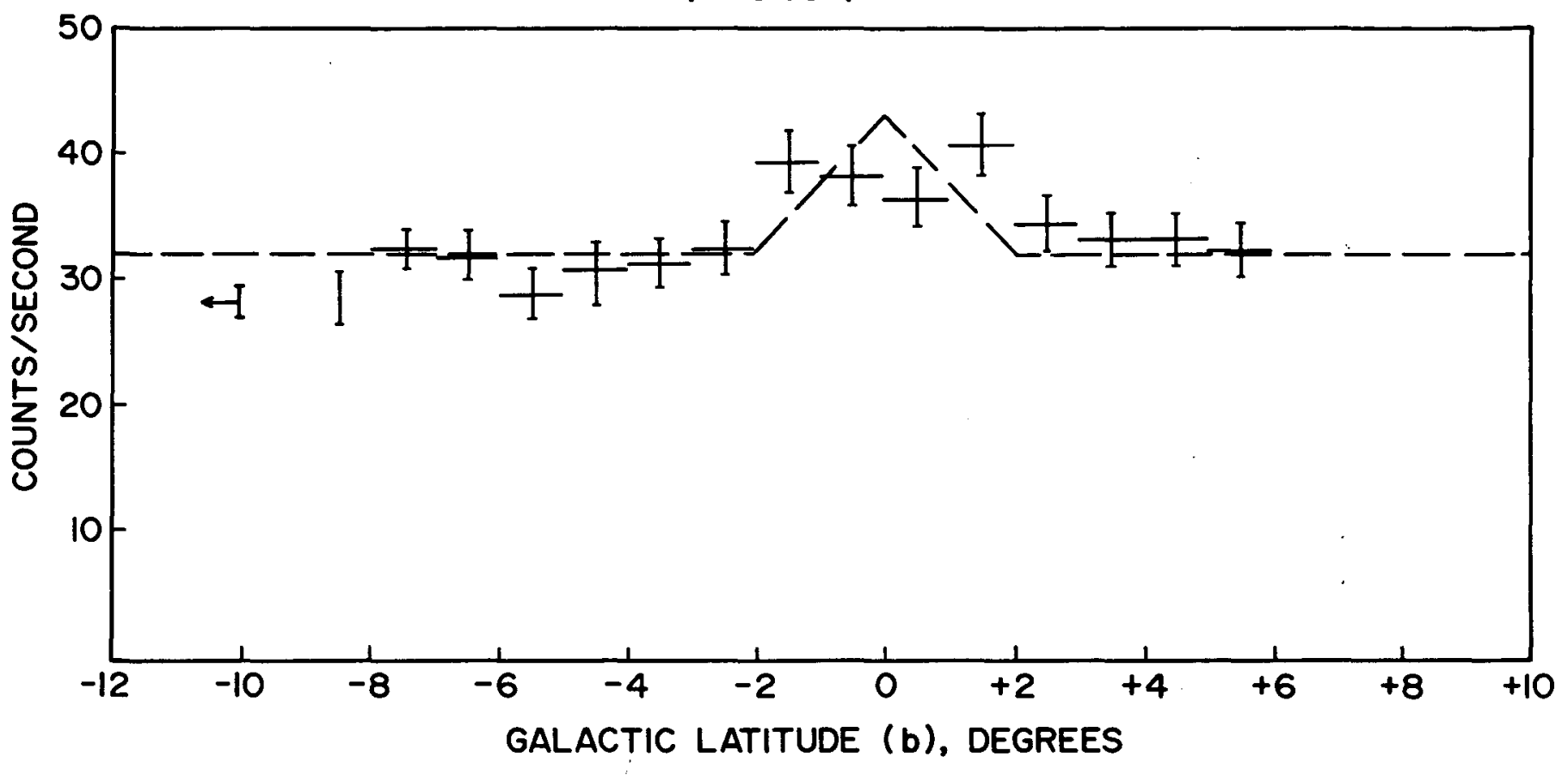

DISK MODEL FIT TO GALACTIC PLANE PROFILE $\left(\ell=61.8^{\circ}\right)$

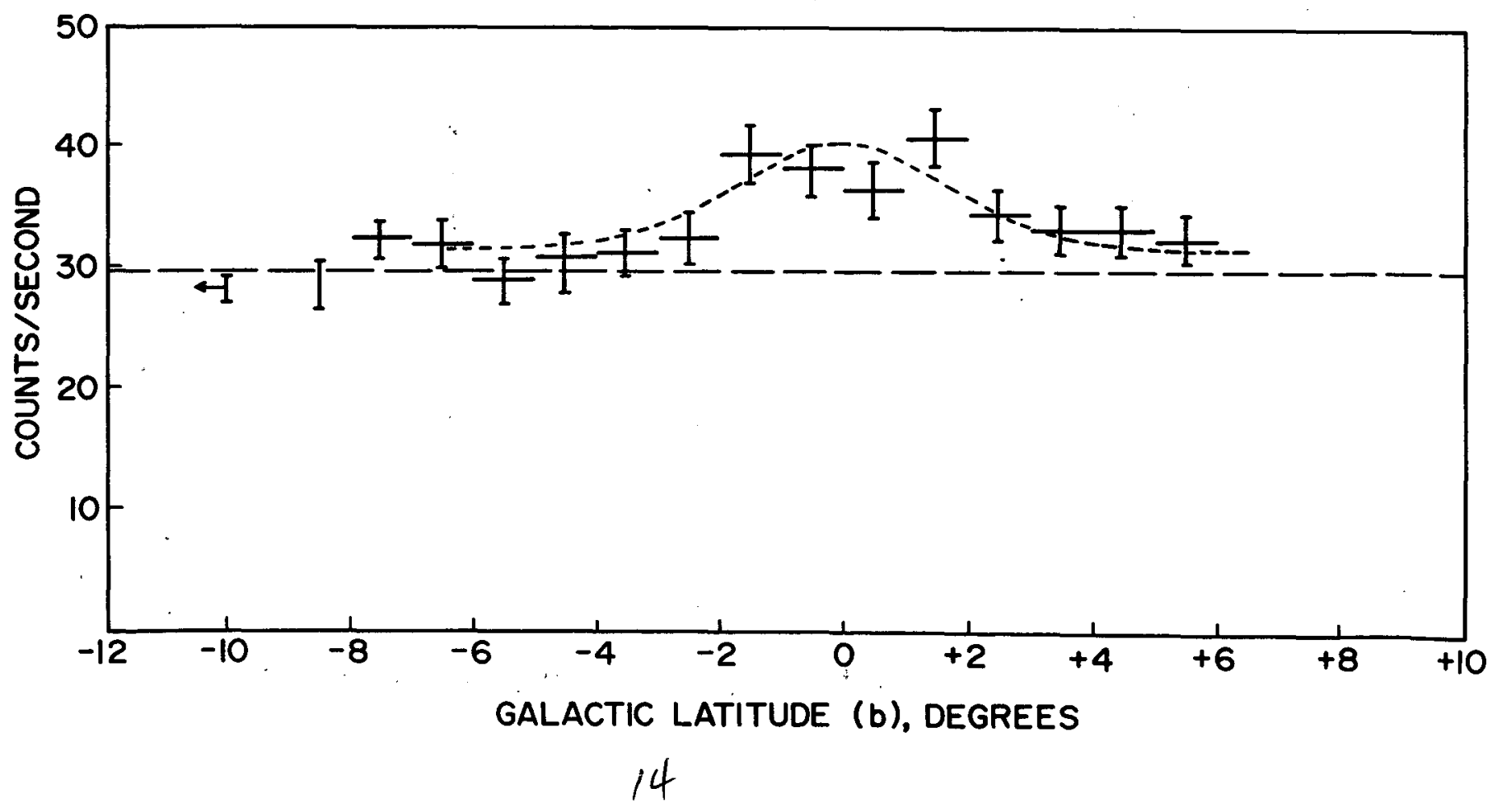



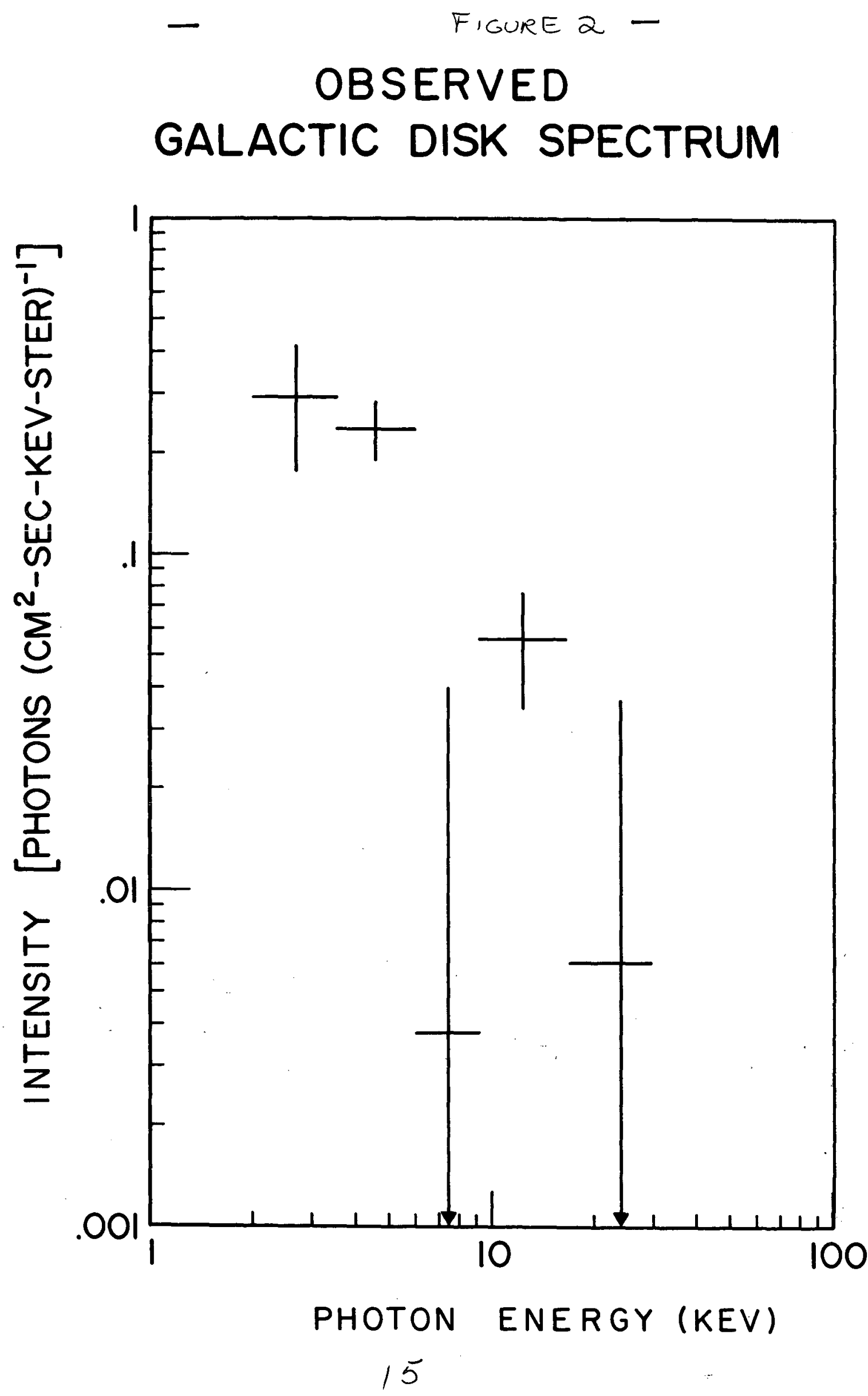